УДК 111.1

DOI 10.18413/2687-0967-2020-47-4-939-950

\title{
О понятии политической реальности
}

\author{
А.И. Швырков \\ Брянский государственный технический университет, \\ Россия, 241035, Брянск, б-р 50 лет Октября, 7 \\ E-mail: aishvyrkov@ rambler.ru
}

\begin{abstract}
Аннотация. В статье сделана попытка сформулировать определение понятия политической реальности. В своих более ранних публикациях автор уже обращался к этому вопросу. Тогда он полагал, что политическая реальность не может и не должна отождествляться с политическими фактами. Скорее, она служит их основой, порождает их, являет себя в них. Хотя с точки зрения автора подобное представление о политической реальности (да и о реальности вообще) является вполне адекватным, нельзя не признать, что для решения большинства задач, с которыми сталкивается политология, его сложность, даже изощренность излишни. Наоборот, определение, в основе которого лежит отождествление политической реальности и фактов, пожалуй, оказывается вполне достаточным. Однако при условии некоторой его коррекции. Такое скорректированное определение политической реальности может выглядеть следующим образом. Политическая реальность - это множество политических фактов, которым соответствует потенциально истинная картина. Картина - это набор утверждений, касающихся некоего политического события, персонажа и т. п. Утверждение истинно, если факт, в нем выраженный, имеет место, ложно - если не имеет. Картина истинна, если истинны составляющие ее утверждения. Новые факты, обнаруженные или выведенные, могут приводить к перестройке картины. Поскольку факты в принципе не могут закончиться, потенциально картина может перестраиваться бесконечно. Поскольку на счет фактов никогда нельзя быть уверенным, что они имеют/имели место, истинность любой картины является лишь возможной, то есть потенциальной именно поэтому в приведенном определении речь идет о потенциально истинной, а не о просто истинной картине.
\end{abstract}

Ключевые слова: реальность, факт, утверждение, картина, потенциально истинная картина.

Для цитирования: Швырков А.И. 2020. О понятии политической реальности. Via in tempore. История. Политология, 47 (4): 939-950. DOI: 10.18413/2687-0967-2020-47-4-939-950.

\section{On the concept of political reality}

\author{
Aleksandr I. Shvyrkov \\ Bryansk State Technical University, \\ 750 let Oktyabrya Boulevard, Bryansk, 241035, Russia \\ E-mail: aishvyrkov@rambler.ru
}

\begin{abstract}
The article attempts to formulate a definition of the concept of political reality. In his earlier publications, the author has already addressed this issue. Then he believed that political reality cannot and should not be identified with political facts. Rather, it serves as their basis, gives rise to them, manifests itself through them. Although, from the author's point of view, such an idea of political reality (and, probably, reality in general) is quite adequate, he cannot but admit that its complexity and even sophistication are superfluous for solving those problems which political science usually deal with. On the contrary, the definition, which is based on the identification of political reality as facts, seems to be quite sufficient. However, subject to some correction. Such an adjusted definition of political reality can look as follows. Political reality is a set of political facts
\end{abstract}


that corresponds to a potentially true picture. A picture is a set of statements regarding a certain political event, character, etc. The statement is true if the fact, expressed in it, takes place, false - if it doesn't. The picture is true if statements, which constitute it, are true. New facts discovered or deduced may lead to a rearrangement of a picture. Since facts cannot run out in principle, a picture can potentially be rearranged infinitely. Since it is never possible to be sure that certain facts took/take place, the truth of any picture is only possible, that is, potential. That's why the definition above speaks about a potentially true but not just true picture.

Keywords: reality, fact, statement, picture, potentially true picture.

For citation: Shvyrkov A.I. 2020. On the concept of political reality. Via in tempore. History and political science, 47 (4): 939-950 (in Russian). DOI: 10.18413/2687-0967-2020-47-4-939-950.

Понятие политической реальности, хотя и употребляемое сплошь и рядом в политологической литературе, тем не менее, как правило, нигде четко не эксплицируется, воспринимается как само собой разумеющееся ${ }^{98}$. Это, впрочем, неудивительно, коль скоро даже в чисто философской литературе попытки прояснить понятие реальности можно пересчитать по пальцам - чаще всего оно «теряется» в/среди таких понятий, как «бытие», «сущее», «существование» и т. п. Пожалуй, единственная серьезная попытка ответить на вопрос «что такое реальность?» с философской точки зрения была осуществлена в книге С.Л. Франка «Реальность и человек» [Франк, 2009].

Тем не менее понятие политической реальности, очевидно, нуждается в прояснении. Хотя бы по формальным основаниям.

Несколько лет назад я предпринял попытку такого прояснения. Причем существенным образом опираясь на идеи Франка (изложенные также в его «Непостижимом»).

Также в своем определении реальности я во многом отталкивался от понятия политического факта. Причем «отталкивался» в буквальном смысле, то есть утверждал, что политическая реальность к ним, фактам, не сводится, что ее нельзя рассматривать как всего лишь синоним совокупности фактов. Предварительные результаты моих размышлений были опубликованы в нескольких статьях (в частности [Швырков, 2014, 2016]).

В общем и целом то определение (или лучше вѝдение) политической реальности, на которое я тогда вышел, представляется мне вполне адекватным и сегодня. Однако должен признать, что работать с подобным определением политологу довольно трудно. Хотя бы потому, что оно слишком «философское».

С философской точки зрения реальность действительно нельзя сводить к фактам или всей их совокупности (в упомянутых статьях я предлагал говорить о том, что реальность «порождает» факты, что мы «черпаем» факты из реальности с помощью теории см. ниже). Однако с другой стороны, когда мы используем термин «реальность» в конкретных науках, такая «точность измерений» нам просто не нужна, она оказывается излишней, а то и неоправданно усложняет построения.

Вообще, необходимость в более глубоком видении реальности, как правило, появляется тогда, когда политолог вынужден действовать на границе политологии и философии (хотя необходимо признать, что такое бывает не так уж редко). В этом случае действительно может возникнуть необходимость в рассмотрении политического факта

${ }^{98}$ Как писал еще в 1968 году Джон Ганнел (John Gunnell), «B сфере политической науки философский вопрос о природе политической реальности... поднимался довольно редко, хотя ответ на него в конечном итоге определяет и обосновывает, что именно наука имеет в виду» [Gunnell, 1968: 166]. Судя по анализу политологической литературы последних десятилетий, ситуация с тех пор мало в чем изменилась. О необходимости более серьезного отношения к основам - в том числе и философским - политологии см., например, [Гаман-Голутвина, 2019]. 
не просто как первичного материала для анализа, а как результата некой интеллектуальной работы.

Другой случай, когда представление о реальности как факте нас уже не может (да, наверное, и не должно) удовлетворить, возникает тогда, когда мы обращаемся к анализу происхождения нашей науки, а также ее основаниям ${ }^{99}$.

Возникновение той или иной науки знаменуется появлением нового типа фактов (см. ниже). То есть некая конкретная наука, как правило, начинается с обособления, выделения некоторых фактов или, точнее, «маркирования» тех или иных фактов особым образом. Причем маркирование этих фактов является внешним по отношению и к фактам, и к самой науке актом.

Когда мы обращаемся к истокам и основаниям той или иной науки, природа этого акта приобретает первостепенное значение, для нас становится важным, каким образом и почему определенные факты приобретают новую «маркировку». И здесь те отношения между реальностью и фактами, те сущностные различия, которые между ними есть и которые я попытался выявить в упомянутых выше статьях, действительно приобретают первостепенное значение. Однако, когда мы сами действуем изнутри науки, вопрос о том, что лежит за теми фактами, с которыми она имеет дело, оказывается почти излишним.

Кто-то мог бы усомниться в том, что та реальность, которую я ассоциирую с фактами, является той же самой реальностью, которая эти факты «порождает». Действительно, не имеем ли мы здесь дело с разнылми понятиями? Хотя этот вопрос, конечно, нуждается в серьезном анализе, думаю, ответ на него будет скорее отрицательным. Позволю себе образный пример, иллюстрирующий данную ситуацию: хотя над водой видна только часть айсберга (причем меньшая), это не значит, что под водой находится какой-то совсем другой айсберг.

Итак, резюмирую. Если не покидать сферу политологии, определение, в основе которого лежит отождествление реальности и фактов, пожалуй, может оказаться вполне достаточным для решения тех задач, с которыми чаще всего приходится иметь дело политологу. Для той «точности измерений», которая здесь необходима, его, такого определения, хватит.

Хотя, конечно, просто сказать, например, что политическая реальность - это «все политические факты», будет не достаточно. Вероятно, даже в этом «простом» случае все не так просто - иначе мы бы давно четко зафиксировали, эксплицировали данное тождество. То есть подобное утверждение должно быть дополнено, уточнено, конкретизировано.

Как раз этим я и собираюсь заняться ниже. Однако сначала еще несколько важных моментов.

Прежде всего, предвосхищая возможные возражения типа того, почему это философ указывает политологам, что им следует понимать под политической реальностью, хочу сказать следующее. А кто же еще, кроме философа, должен этим заниматься?! Вопрос о реальности - политической, физической, просто реальности - это вопрос фундаментальный, основополагающий. Вопрос о реальности, которую изучает и к которой апеллирует конкретная наука - суть вопрос о том, что выходит за рамки этой науки. Ее языковых, методологических и прочих средств просто не хватит для ответа на него. Поэтому философия за него и берется, должна браться. Ибо подобные вопросы как раз и определяют одну из основных сфер ее деятельности.

Другой вопрос: почему не начать с собственно реальности, «реальности вообще». Так, чтобы под результирующее определение подпадали любые «частные» реальности, в том числе и политическая. Здесь я должен апеллировать к случайным

${ }^{99}$ На это как раз указывал Дж. Ганнел в цитировавшейся статье. 
обстоятельствам, связанным с моими научными интересами. Дело в том, что «исторически» вопрос о реальности для меня возник именно в связи с политологическими изысканиями, так сказать, в ходе философского вояжа в политологическую (и даже политическую) область. Именно поэтому я намеренно «зауживал» свой горизонт.

Однако я не имею ничего против, если кто-то захочет показать, что то определение, которое будет представлено ниже, можно расширить на реальность как таковую, реальность «вообще».

Наконец последняя вводная.

В своих построениях я в значительной степени буду опираться на наработки аналитической философии.

Хотя аналитическая философия, как кажется, во многом достигла своих пределов [Джохадзе, 2016], она тем не менее успела создать достаточно развитый аппарат (в том числе терминологический), который по крайней мере позволяет говорить о том клубке, узле проблем, которые порождаются (или составляют) отношениями фактов и реальности, не впадая при этом в откровенные спекуляции и не выходя в область совершенного умозрения ${ }^{100}$. Причем позволяет говорить лучше, чем любая другая теоретическая система.

Во многом разделяя скепсис по поводу возможности создания аналитической политической философии [Павлов, 2010], я все же решусь утверждать, что в отдельных вопросах - в частности таких, как тот, который исследуется в данной статье, - аналитическая философия может оказаться достаточно эффективной. Сформулирую подругому. Хотя аналитическая философия вряд ли способна ответить на вопрос о том, что такое реальность (точнее, ответить на этот вопрос более глубоко, чем какие-то иные философские концепции, - того же С. Франка), она по крайней мере может обеспечить возможность для конкретных наук (в нашем случае - политологии) пользоваться некоторыми понятиями - той же политической реальности - не «повисая в воздухе», не апеллируя к некой их «интуитивной понятности». Впрочем, это не удивительно, поскольку аналитическая философия изначально стремилась к максимально точным, максимально конкретным, верифицируемым формулировкам, вообще к максимальному наукоподобию (в лучшем значении этого слова). Другое дело, конечно, насколько ей это удалось, точнее, действительно ли подобный путь - это магистральный путь философии.

Далее. Думаю, есть смысл привести краткое описание моего первоначального, не раз уже упоминавшегося выше понимания реальности. Отчасти для того, чтобы дать читателю более наглядное представление о тех проблемах, о которых шла речь в связи с возможностью двух определений политической реальности, отчасти для того, чтобы ему было проще проверить обоснованность того «поворота на $180^{\circ}$ » (по сравнению с моими более ранними публикациями), который я собираюсь совершить.

Впервые это определение было сформулировано в статье «Об отношениях между политической теорией и реальностью», опубликованной в журнале «Полития» [Швырков, 2014]. В этой статье, как следует из ее названия, я попытался обрисовать то, каким образом политическая теория соотносится с политической реальностью.

Политическую теорию я понимал и понимаю вполне традиционно как комплекс вертикально и горизонтально связанных общих утверждений, толкующих политические факты.

${ }^{100}$ См., например, [Витгенштейн, 2009]. Современный терминологический аппарат, см. Wetzel T. States of Affairs. Stanford Encyclopedia of Philosophy. URL: https://plato.stanford.edu/archives/win2016/entries/states-of-affairs/ (accessed: 10.11.2019). 
В случае политики в качестве фактов могут рассматриваться, например, результаты голосования, публичные заявления того или иного политика, те или иные политические решения и действия (например, объявление войны, политически мотивированное экономическое эмбарго, вхождение политической партии в коалицию) и т. п. Более строго, под политическим фактом я буду понимать некое действительное или предполагаемое политическое событие. Факт, как правило, выражается с помощью некоторого утверждения.

В упомянутой статье я также предложил не спешить отождествлять факты и реальность ${ }^{101}$. Ближайшая - но не единственная - причина этого та, что факты никогда не существуют, что называется, сами по себе, факты - это всегда результат некоей предварительной работы: ведь нам же надо было хотя бы разграничить политические, экономические, физические, исторические и т. д. факты. То есть, факты - это уже некий полуфабрикат ${ }^{102}$.

Как политический факт возможен только благодаря, в присутствии политической теории. Факт немыслим в отрыве от некоего концептуального поля, обеспечиваемого, задаваемого теорией. То есть до того как появляется соответствующая теория, политических фактов не существует ${ }^{103}$. Тем не менее сказанное вовсе не означает, что политическая теория появляется до политического факта или независимо от него. Думаю, речь следует вести о неком сдвиге в сознании, порождающем и политическую теорию, и политические факты. И снова-таки сам по себе этот сдвиг не обязательно должен проявиться в создании некой политической теории. Часто он приводит лишь к тому, что некие ранее созданные теории начинают восприниматься как политические ${ }^{104}$.

Важно отметить, что, как только возникает такой сдвиг, мыслить те факты и те теории, которые раньше считались неполитическими, - а теперь, после этого сдвига, считаются политическими - как неполитические становится очень трудно, то есть становится трудно представить, что когда-то эти факты и теории политическими не являлись.

Однако раз факты - это не первое, не изначальное, значит, за ними должно быть что-то еще, что-то, откуда мы «черпаем» факты (я бы сказал - с помощью теории). Факты, таким образом, оказываются представлением чего-то, скрывающегося за ними. И как раз то, что скрывается за фактами (или наоборот, являет себя в фактах), я и назвал реальностью.

Реальность, таким образом, суть нечто, лежащее в основе фактов (вещей, феноменов, явлений и т. д.), нечто, выражаясь метафорически, их порождающее. Эта реальность бесконечна, непостижима, неисчерпаема. Она подобна Богу средневековых философов или Непостижимому С. Франка (или его же реальности [Франк, 1990, 2009]).

Однако при всем при этом, пожалуй, нельзя сказать, что она абсолютно недоступна. Пожалуй, нет ничего доступней реальности - ведь она всегда «здесь», всегда «рядом», всегда «вокруг».

${ }^{101}$ Как кажется, к такому отождествлению был склонен Э. Дюркгейм [Дюркгейм, 1996]. Следует заметить, что чаще всего такое представление о реальности в политологических текстах не эксплицируется, а подразумевается (то есть выявить его можно только по контексту, в котором употребляется соответствующие термины).

102 «...факты нельзя... просто воспринимать, они должны быть установлены... Факт не существует независимо от того, замечает ли его кто-нибудь или нет; скорее, факт - это итог размышлений» [Walsh, 1970: 77].

${ }^{103}$ Ср. с появлением безумия у М. Фуко [Фуко, 2010].

104 Думаю, именно это произошло, например, с теориями Платона или Аристотеля. До этого их «политические» произведения могли рассматриваться только лишь как, например, учения о справедливом общественном устройстве или просто о справедливости. 
Однако почему же в упомянутой статье я говорю об отношениях теории и реальности? Ведь из сказанного в предыдущих абзацах выходит, что теория имеет дело исключительно с фактами. Реальность же я понимаю как отличную от этих последних.

Теория - суть набор символов. Не будь языка, не будь письменности, ни о какой теории не могло бы быть и речи. Факты, как уже было сказано, не могут существовать без теории, факт, чтобы действительно появиться, должен быть описан. По сути, момент описания факта (причем - в терминах теории) и есть момент его рождения. Что касается реальности - по крайней мере, в моем понимании, - то она принципиально невербальна. То, что выражено в словах - уже не реальность. Соотносить можно только то, что имеет одну природу. Поэтому понятно, что теории всегда соотносятся только с фактами. Однако.

Однако теории не даны нам как нечто готовое (точно так же, как и факты). Теории творятся. Творятся же они познающим субъектом как ответ на реальность, реальность, воспринимаемую им, кстати сказать, непосредственно, во всей ее полноте (то есть не как совокупность фактов). Другими словами, хотя теория действительно соотносится с фактами (можно сказать, что и факты, и теории «состоят» из одной «материи»), относится она всегда к реальности.

Кратко напомнив свое первоначальное определение политической реальности, попытаюсь теперь сформулировать мое новое ее понимание.

Факт я буду понимать так же, как и раньше. Факт может иметь место, а может не иметь места. Если факт имеет место, то соответствующее утверждение (то есть утверждение, выражающее факт) будет истинным. Если факт не имеет места, соответствующее утверждение будет ложным. Например, если США действительно ввели войска в Сирию, значит, факт ввода войск США имел место, а утверждение «США ввели войска в Сирию» будет истинным (утверждения, как это принято в аналитической философии, я буду брать в кавычки).

Установить факт - значит установить, что факт имеет место.

Картиной назовем набор утверждений, касающихся некоего события, персонажа и т. п. Может быть картина Уотергейтского скандала, протестов на Болотной площади, связи Билла Клинтона с Моникой Левински и т. д., и т. п.

Картина истинна, если истинны составляющие ее утверждения.

Реальность события, персонажа, вокруг которого выстраивается картина, не является заранее известной. То есть факт события, факт существования персонажа не обязательно должен быть установленным.

Некоторые утверждения, составляющие картину, могут быть истинными, некоторые - ложными. Факты, соответствующее первым, имеют место, вторым не имеют.

Картина не является раз и навсегда заданной. Она может перестраиваться в связи со вновь обнаруженными фактами.

Некоторые утверждения картины могут противоречить другим утверждениям. Например, «некто был на месте убийства президента Кеннеди в момент убийства» и «некто не был на месте убийства президента Кеннеди в момент убийства». И то и другое утверждения могут быть основаны на показаниях свидетелей. В этом случае (наличия в картине противоречивых утверждений) могут производиться дополнительные изыскания. Рассмотрим это подробнее.

Предположим, мы захотим проверить, имеет место тот или иной факт или нет. Конечно, мы может попытаться это, что называется, «вычислить». То есть установить, имеет место факт или нет, чисто спекулятивным путем, с помощью анализа соответствующих утверждений (основываясь в первую очередь на законах формальной логики). Однако это возможно лишь в очень ограниченном числе случаев, 
обычно тогда, когда у нас есть избыток информации (причем значительная ее часть является такой, в которой мы уверены). Поэтому чаще всего мы просто начинаем «копать», то есть искать факты, которые бы смогли подтвердить или опровергнуть данный факт. Например, ищем свидетелей, документы и т. п. Понятно, что в ходе таких поисков мы можем снова попасть в ситуацию, подобную той, которая заставила нас эти поиски предпринять. Тогда мы будем искать факты, подтверждающие факты, подтверждающие факты, подтверждающие факты и т. д., вообще говоря, до бесконечности. Важно в этом контексте то, что факты можно найти всегда, то есть невозможно представить себе случай, когда факты вдруг «закончатся» - сама эта фраза звучит нелепо.

Однако бесконечности у нас нет ни в плане времени, ни в плане ресурсов. Поэтому рано или поздно мы свои поиски остановим. Но, естественно, не просто так, не в произвольной точке (если, конечно, не случится чего-то экстраординарного). Для остановки поисков существует несколько критериев:

1. Картина события «сложится» таким образом, что существенных видимых противоречий, неувязок, необъясненных фактов в ней не останется. В политике такое бывает крайне редко, поскольку политические события обычно довольно сложны с точки зрения числа их участников, наличия специально скрываемых обстоятельств, возможности сознательной подтасовки фактов, манипуляций и т. п.

2. Мы зайдем в тупик. В том смысле, что у нас никак не будет возможности «собрать все в кучу», то есть, по сути, нарисовать целостную картину. Те или иные факты вроде бы будут относиться к тому или иному событию, персонажу и т. п., но соединить их таким образом, чтобы картина стала связной, логичной и проч., мы будем не в состоянии.

3. У нас не будет физической возможности установить тот или иной факт. Например, для проверки того или иного факта нам необходимо будет попасть на некую военную базу, в архив и т. п., однако у нас не будет для этого допуска.

Выше речь шла в основном о фактах, которые могут быть названы первичными (фактами первого порядка, базовыми и т. п.). Такие факты могут быть установлены непосредственно. Однако существуют и такие факты, которые устанавливаются в результате анализа, интерпретации первичных фактов. Назовем их вторичными (фактами второго порядка, выводными фактами и т. п.).

Получение вторичных фактов, очевидно, предполагает проведение некой интеллектуальной работы. Она основана на использовании интуиции, опыта, теории.

Существует ли принципиальная разница между первичными и вторичными фактами? Или вся разница - только в способе их установления? Чаще всего мы имеем дело со вторым случаем, то есть первичные и вторичные факты оказываются, так сказать, фактами одной материи. Однако дать окончательный ответ на этот вопрос еще предстоит ${ }^{105}$.

В качестве примера вторичного факта рассмотрим «гибридную войну», якобы идущую между Россией и Украиной.

Некоторые политики, эксперты и проч. утверждают, что факт такой войны имеет место. Другие - что нет. При этом что такое гибридная война вроде бы секретом не является. Определение ее легко найти в интернете, научной литературе. В чем же тогда дело? Почему существуют противоположные мнения по поводу этого факта (гибридной войны)?

Есть два фактора, ответственных за это. Первый - собственно факты (которые подтверждали бы или опровергали факт гибридной войны). Часть этих фактов - первичные факты, часть - вторичные.

105 Возможно, что разделение на первичные и вторичные факты является чисто cumyamuвныlм. 
Обнаружение первичных фактов, очевидно, сопряжено со значительными трудностями, часто чисто физического плана (например, выезд в тот или иной населенный пункт может быть невозможен из-за боевых действий). Обнаружение вторичных фактов (точнее, формирование соответствующих утверждений) зависит от интуиции, опыта экспертов, теории, которой они располагают.

Второй фактор - это как раз теория.

Выше я сказал, что в литературе можно легко найти определение гибридной войны. Однако это определение не достаточно для того, чтобы точно определить, в каких именно случаях гибридная война имеет место, а в каких - нет. То есть оно слишком общее, слишком расплывчатое. Для того чтобы его уточнить, конкретизировать, необходимо, по сути, создать целую теорию. Причем такую, которую бы признали большинство ученых и экспертов. Сегодня такой теории нет.

Почему факт гибридной войны является вторичным? Потому что для его установления, как мы видим, необходимо собрать множество других фактов, интерпретировать их на основе теории и т. д.

Установление вторичного факта, вообще говоря, часто оказывается делом довольно непростым.

Эти предварительные разъяснения позволяют сформулировать понятие (новое) политической реальности следующим образом.

Под политической реальностью я понимаю множество политических фактов, которым соответствует потенциально истинная картина.

В этом определении, очевидно, самый «проблемный» момент - это фраза о потенциальной истинности картины. Почему речь идет именно о потенциально истинной картине, а не о просто истинной?

Когда речь заходит о политике, тех или иных политических событиях, мы, как правило, верим, что всегда или почти всегда есть кто-то, кто точно знает, «как было дело», кто наверняка знает, какие факты имели/имеют место, а какие - нет ${ }^{106}$. То есть что есть кто-то, кто знает «истину».

Ясно, что речь идет о людях, которые либо были непосредственно вовлечены в те или иные события (например, политики), либо «наблюдали» за участниками этих событий, так как это входит в их «служебные обязанности» (например, представители спецслужб), либо их «расследовали» (например, журналисты). Ведь, в конце концов, должен же какой-то политик точно знать, говорил он что-то другому политику в частной беседе или нет (случай, когда такой политик находится под действием психотропных веществ, мы здесь не рассматриваем) ${ }^{107}$.

Мы выстраиваем картину того или иного политического события, опираясь на знание того / веру в то, какие факты имели место (знание о том / вера в то, какие факты места не имели, то есть негативное знание, очевидно, имеет для построения картины гораздо меньшее значение) ${ }^{108}$.

Эти два момента (то есть что картина выстраивается на знании того / вере в то, что некие факты имели место, и что есть кто-то, кто точно знает, какие

${ }^{106}$ По крайней мере, в отношении некоторого количества первичныл фактов. В отношении вторичных фактов этого утверждать нельзя, так как их вывод зависит от политической теории, а в отношении такой теории (ее истинности) действуют все те ограничения, которые действуют в отношении любой теории. В частности те, в соответствии с которыми любая теория может - и должна! - быть сфальсифицирована.

107 Здесь, очевидно, можно усмотреть сходство с преступлениями. Однако между политическими событиями и преступлениями есть и существенное отличие - в случае с первыми для сокрытия их «истинных» причин, обстоятельств, с ними связанных, часто задействуются очень серьезные ресурсы. В случае со вторыми это случается гораздо реже (а если и случается, то чаще всего здесь как раз оказывается замешана политика и/или политики).

108 Для выражения моей мысли здесь лучше всего подошло бы английское слово «believe». 
факты имели место) порождают если не веру, то хотя бы надежду на принципиальную возможность получения представления об истинной картине того или иного события ${ }^{109}$.

Причем, как кажется, существует иллюзия, что построение истинной картины не сопряжено с непреодолимыми трудностями (по крайней мере, принциипиально непреодолимыми) - ведь если кто-то знает, какие факты, относящиеся к тому или иному событию, имели место, а какие - нет, почему бы и нам этого, в конце концов, не узнать. Ведь если тот или иной политик сегодня находится у власти и может отгородиться от нашего «интереса» охраной и высокими заборами, это не значит, что позднее его нельзя будет «допросить» (иногда и без кавычек) «со всей строгостью».

Я уже не говорю о возможности получить доступ к документам, видеозаписям, фотографиям, архивным материалам и проч. Пусть не сегодня, когда страной правит «хунта» и доступ ко многим данным закрыт, так завтра, когда хунта «падет».

Да, подумает кто-то, мы действительно далеко не всегда можем получить доступ к тем или иным людям или документам, но в теории-то это возможно. Да, может быть, я этого и не узнаю, но другие-то могут узнать («вообще», «в принципе»). Если не мы, то, возможно, другое поколение. Если те или иные документы мы не можем прочитать сегодня, потому что у нас нет доступа к архивам, их прочтут наши потомки. И т. д., и т. п.

Казалось бы, история только подкрепляет подобные предположения/надежды. Вспомним хотя бы Нюрнбергский процесс.

Можно без особого труда показать, что легкость здесь кажущаяся. Что всегда можно найти основания для того, чтобы поставить под сомнение даже самый очевидный факт, что любой документ можно подделать, что любую информацию (даже достоверную) можно представить таким образом, что картина того или иного события окажется бесконечно далека от истинной, и т. д., и т. п.

К тому же препятствия на пути исследователя (как то «охрана» и «высокие заборы» и т. п.), которые были упомянуты выше, на самом деле не являются всего лишь техническими, временными, случайными препятствиями, они сущностны, принципиальны для политической сферы. Хотя ты можешь находиться в метре от политика, можешь даже попытаться его убить (и даже убить) - что не так уж и редко происходило и происходит, - это вовсе не означает, что у тебя есть или когда-либо гарантированно появится к нему достуn, что ты можешь действительно узнать, что он думает или хотя бы говорил. То же самое касается документов и проч. ${ }^{110}$

Это «наблюдение» можно распространить на любые факты - как первичные, так и вторичные. Ведь поскольку на самом деле мы не можем «добраться» (то есть установить, что они имели/имеют место) даже до тех первичных фактов, о которых кому-то доподлинно известно, что они имели/имеют место, эти факты уравниваются в своем статусе с теми, о которых такого сказать нельзя в принципе. То есть все первичные факты (а уж тем более вторичные) оказываются окончательно неустанавливаемьли при том, что по аналогии с фактами первого типа (о которых кто-то знает, что они имели/имеют место), «в принципе», то есть потенциально, их установить можно. К установлению их можно бесконечно приближаться, но так и не приблизиться вплот-

${ }_{110}^{109}$ На этом же во многом основана и вера в теорию заговора.

${ }^{110}$ Недоступность, закрытость политики - это вообще ее сущностная черта. А иначе откуда бы взялись все эти призывы к «открытости», «транспарентности» и т. п.? 
ную (здесь напрашивается очевидная аналогия с определением математического предела).

Однако при всем при этом, какие бы основания не доверять тем или иным сведениям я или кто-то другой ни приводил, не существует логических оснований считать, что истинная картина того или иного политического события является принципиально невозможной. По крайней мере, вполне законно утверждать, что некто может составить себе (и даже описать, зафиксировать на каком-либо носителе и сделать достоянием гласности) представление о каком-либо политическом событии, которое действительно является истинным, но при этом не знать, что это представление истинно. То есть некая картина может быть истинной, но при этом о том, что она истинна, никто знать не будет (считая эту картину лишь вероятной).

Другими словами, у нас есть все основания утверждать, что даже если в той картине, которая у нас есть сейчас, мы и не уверены (например, по тем основаниям, на которые я указывал выше - закрытость политической сферы, возможные подтасовки, подлоги, искажения и проч.), это вовсе не означает, что истинная картина не может существовать в принциипе. То есть, что потенциально она не может быть получена.

Подытожу.

Любая картина может детализироваться до бесконечности. То есть, мы можем находить и находить первичные факты, относящиеся к ней, можем без конца переформулировать вторичные факты и т. д., и т. п. ${ }^{111}$ При этом какую бы картину мы ни получили, мы никогда не будем до конца уверены, что она истинная. То есть никакую «окончательную картину» мы не способны получить в принципе (сомневаясь даже в той, которая действительно истинной является).

Дело об убийстве президента Кеннеди или такие сравнительно недавние дела, как дело о «вмешательстве» России в президентские выборы в США, «дело Трампа» (расследовавшееся прокурором Мюллером) служат прекрасными иллюстрациями того, о чем я говорю. Потенциально такие дела могут расследоваться годами, десятилетиями. Единственное принципиальное ограничение длительности подобных расследований связано лишь с конечностью нашего существования.

И при всем при этом у нас нет логических оснований утверждать, что «истинная картина» не может существовать в принципе. То есть у нас нет оснований не верить, что рано или поздно мы станем обладателями такой картины.

Момент ускользания реальности, являющийся ключевым в приведенном определении, очевидно, роднит его с ранее приведенной трактовкой этого понятия ${ }^{112}$. При этом данное определение является более конкретным, кратким, операционализируемым. Понятным, наконец (надеюсь). Ясно также, что эти его характеристики были достигнуты главным образом за счет существенного сужения «базы» определения: введения ряда явных и неявных ограничений, условий, допущений.

\footnotetext{
111 Аналогия с фракталами!

112 Именно поэтому я удовлетворен этим определением и могу «смириться» с тем, что оно является более узким, чем первоначальное.
} 


\section{Список литературы}

1. Витгенштейн Л. 2009. Логико-философский трактат. М., Наука, 133 с.

2. Гаман-Голутвина О.В. 2019. Преодолевая методологические различия: споры о познании политики в эпоху неопределенности. Полис. Политические исследования, 5: 19-42. DOI: 10.17976/jpps/2019.05.03.

5: $1-15$.

3. Джохадзе И. 2016. Аналитическая философия сегодня: кризис идентичности. Логос,

4. Дюркгейм Э. 1996. Метод социологии. В кн.: Западноевропейская социология XIX -начала XX веков: Тексты. М., Международный университет бизнеса и управления: 256-309.

5. Павлов А.В. 2010. Аналитическая политическая философия? Политическая концептология, 2: 175-184.

6. Франк С.Л. 1990. Непостижимое. В кн. Франк С.Л. Сочинения. М., Правда: 183-559.

7. Франк С.Л. 2009. Реальность и человек: метафизика человеческого бытия. Минск, Белорусская Православная Церковь, 560 с.

8. Фуко М. 2010. История безумия в классическую эпоху. М., АСТ МОСКВА, 698 с.

9. Швырков А.И. 2014. Об отношениях между политической теорией и реальностью. Полития, 4: 71-85.

10. Швырков А.И. 2016. Политическая теория, дискурс и реальность: предварительный анализ взаимоотношений. Полис. Политические исследования, 5: 66-79. DOI: 10.17976/jpps/2016.05.06.

11. Gunnell John G. 1968. Social Science and Political Reality: The Problem of Explanation. Social Research, 1: 159-201. $215 \mathrm{p}$.

12. Walsh W.H. 1970. An Introduction to the Philosophy of History. London, Hutchinson,

\section{References}

1. Vitgenshteyn L. 2009. Logiko-filosofskiy traktat [Logical and philosophical treatise]. M., Nauka, 133 p. (in Russian).

2. Gaman-Golutvina O.V. 2019. Bridging Methodological Differences: The Policy Cognition Controversy in an Age of Uncertainty. Polis. Political Studies, 5: 19-42. DOI: 10.17976/jpps/2019.05.03 (in Russian).

3. Dzhokhadze I. 2016. Bridging Methodological Differences: The Policy Cognition Controversy in an Age of Uncertainty. Logos, 5: 1-15. (in Russian)

4. Dyurkgeym E. 1996. Metod sotsiologii [Method of sociology]. In: Zapadnoyevropeyskaya sotsiologiya XIX - nachala XX vekov: Teksty [Western European sociology of the XIX - early XX centuries: Texts]. M., Mezhdunarodnyy universitet biznesa i upravleniya: 256-309 (in Russian).

5. Pavlov A.V. 2010. Analytical Political Philosophy? Political conceptology, 2: 175-184 (in Russian).

6. Frank S.L. 1990. Nepostizhimoye [Incomprehensible]. In: Frank S.L. Sochineniya [Frank S.L. Texts]. M., Pravda: 183-559 (in Russian).

7. Frank S.L. 2009. Real'nost' i chelovek: metafizika chelovecheskogo bytiya [Reality and Man: Metaphysics of Human Being]. Minsk, Belorusskaya Pravoslavnaya Tserkov', 560 p. (in Russian).

8. Fuko M. 2010. Istoriya bezumiya v klassicheskuyu epokhu [The history of madness in the classical era]. M., AST MOSKVA, 698 p. (in Russian).

9. Shvyrkov A.I. 2014. On the relationship between political theory and reality. Polity, 4: 71-85 (in Russian).

10. Shvyrkov A.I. 2016. Political Theory, Discourse and Reality: A Preliminary Analysis of Relationships. Polis. Political Studies, 5: 66-79. DOI: 10.17976/jpps/2016.05.06 (in Russian).

11. Gunnell John G. 1968. Social Science and Political Reality: The Problem of Explanation. Social Research, 1: 159-201.

12. Walsh W.H. 1970. An Introduction to the Philosophy of History. London, Hutchinson, 215 p. 
Via in tempore. История. Политология. 2020. Том 47, № 4 (939-950)

Via in tempore. History and political science. 2020. Volume 47, № 4 (939-950)

ИНФОРМАЦИЯ ОБ АВТОРЕ

Швырков Александр Иванович, кандидат философских наук, доцент кафедры гуманитарных и социальных дисциплин Брянского государственного технического университета, г. Брянск, Россия.

\section{INFORMATION ABOUT THE AUTHOR}

Aleksandr I. Shvyrkov, PhD, Associate Professor of the Department of Humanities and Social Disciplines, Bryansk State Technical University, Bryansk, Russia 